Journal of

Accident and

Emergency

Medicine 1994

11, 257-258

\title{
Multiple carpometacarpal dislocations
}

\author{
A. KUMAR \& D.B. OLNEY \\ Accident and Emergency Department, Frimley Park Hospital, Frimley, Camberley, Surrey
}

\section{SUMMARY}

We present a case of dislocations of the carpometacarpal joints without associated fractures. Although carpometacarpal injuries are relatively uncommon, it is rare for multiple carpometacarpal dislocations to occur without associated fractures. The injury is difficult to diagnose because of swelling of the hand. A lateral radiograph of the wrist has been found to be mandatory to its precise diagnosis if suspected.

In the case presented here early diagnosis and closed manipulation in the accident and emergency (A\&E) department resulted in a good outcome.

Key words: carpometacarpal dislocations, diagnosis, investigation, management, radiographs

\section{CASE REPORT}

A 20-year-old construction worker presented to the A\&E department complaining of pain in his right hand and inability to move his fingers. He stated that he had punched a wall in anger. He admitted to having consumed alcohol.

On examination there was swelling on the dorsum of his right hand. Active movements were not possible due to pain but limited passive movement of the fingers were present. There was no neurovascular deficit in the fingers. A provisional diagnosis of fracture of the metacarpals was made. The routine postero-anterior and oblique radiograph of the hand did not show any fracture but made us suspicious of an injury at the carpometacarpal joints (Fig. 1). A lateral radiograph of the wrist was requested.

The lateral radiograph of the wrist clearly demonstrated carpometacarpal dislocations of the index, middle, ring and little fingers but no fractures were seen (Fig. 2). The dislocations were easily reduced by linear traction of the fingers under axillary nerve block. The reductions were stable. A below elbow cast with the wrist in a neutral position was applied. A check radiograph showed a satisfactory reduction.

The patient was reviewed after 1 week when he was comfortable in his cast. A further 2 weeks later he returned for follow-up having removed his own cast. He had a full range of painfree movements in his hand and wrist.

\section{DISCUSSION}

Two series of carpometacarpal injuries have been reported recently. ${ }^{1,2}$ Both series confirm the points highlighted by the case presented here.

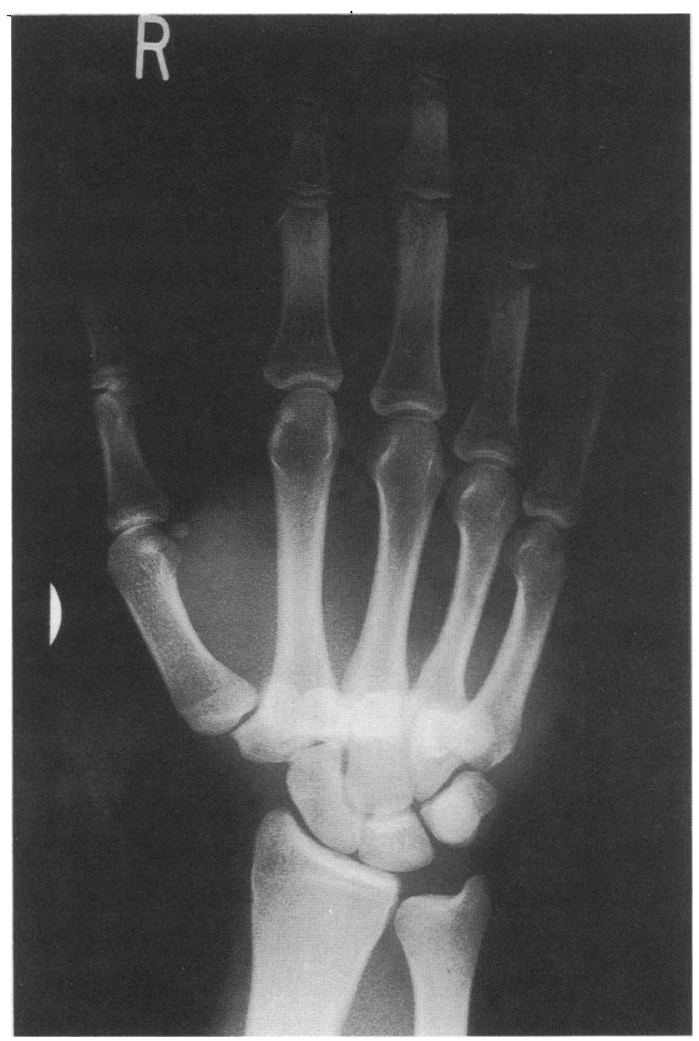

Fig. 1. Postero-anterior radiograph of the hand showing dislocations of the carpometacarpal joints of all the fingers.
Frimley, Camberley, Surrey GU16 5UJ, UK 

A. Kumar \&
D. Olney

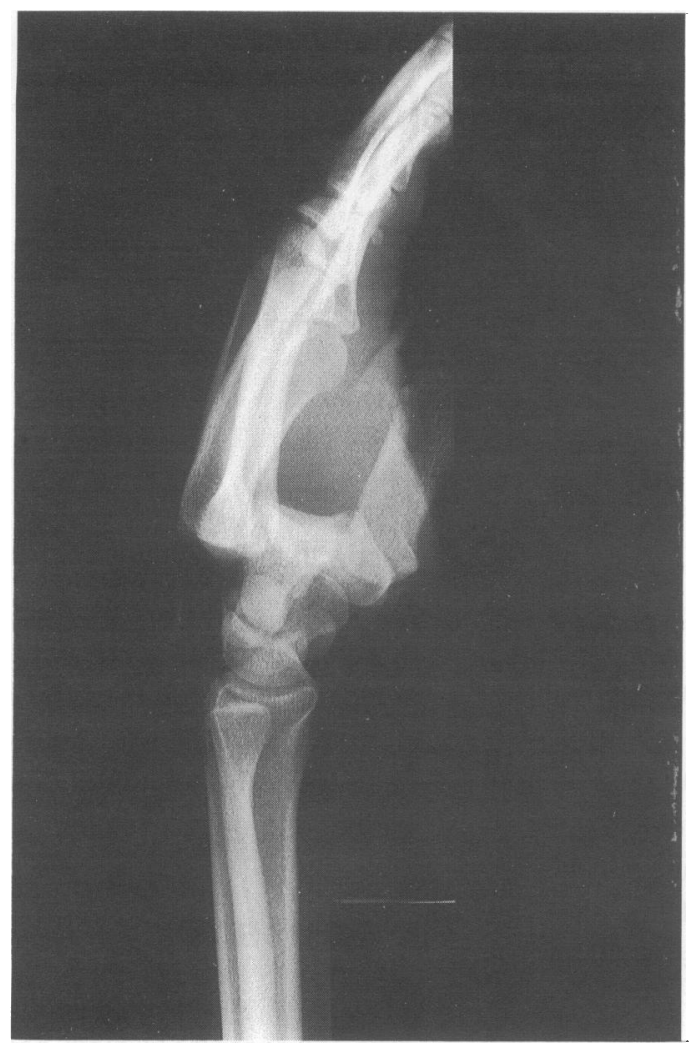

Fig. 2. Lateral radiograph of the hand showing dislocations of the carpometacarpal joints of all the fingers.

Early recognition of this injury in the A\&E department is vital to ensure a satisfactory outcome. Late diagnosis occurs either in cases of multiple injury (as an overlooked injury or not found as an injury at all) or when a lateral radiograph of the wrist has not been taken in suspicious cases of carpometacarpal injury. ${ }^{1,3}$

If associated fractures are seen, reduction may be unstable and may need open reduction and fixation. Early closed reduction in the A\&E department is more likely to produce stable reduction and a satis- factory result. Delay in recognition of this injury is likely to result in the need for open reduction and fixation. An unsatisfactory functional result is then likely.

In this patient, prompt diagnosis of carpometacarpal dislocations of four fingers without associated fractures allowed a closed reduction which was stable. Early return of function to the hand can be expected in this situation.

In normal radiographic practice postero-anterior and oblique views are produced of the hand but it is recommended that a lateral radiograph of the wrist is also taken when there is clinical suspicion of injury in the region of the carpometacarpal joints. This will help inexperienced doctors in A\&E departments to diagnose dislocations of these joints. Early closed reduction by trained doctors should be attempted as a stable reduction is possible especially if there is no associated fracture.

Perhaps, as part of the normal radiographic examination of the proximal part of the hand, a true lateral radiograph of the wrist should always be included?

\section{ACKNOWLEDGEMENT}

I am very grateful to Mr R.J. Partridge, Consultant in the Accident and Emergency Department at Frimley Park Hospital for his guidance.

\section{REFERENCES}

1. De Beer J.D., Maloon S., Anderson P., Jones G. \& Singer M. (1989) Multiple carpometacarpal dislocations Journal of Hand Surgery 14B, 105-108.

2. Lawlis J.F. \& Gunther S.F. (1991) Carpometacarpal dislocations. Journal of Bone and Joint Surgery 73A, 52-59.

3. Parkinson R.W. \& Paton R.W. (1992) Carpometacarpal dislocation: an aid to diagnosis. Injury 23, 187-188. 